\title{
Propuestas de políticas públicas para la prevención de la violencia y el delito en Acapulco, Guerrero 2018*
}

\section{Proposals for public policies for the prevention of violence and crime in Acapulco, Guerrero 2018}

\author{
Javiera Donoso Jiménez ${ }^{* *}$ Pierre Antoine Delice ${ }^{* *}$
}

\begin{abstract}
* Este artículo se enmarca en el tercer entregable del tercer año del Proyecto 1200 "Ciudadanía y violencias en el estado de Guerrero" del programa Cátedras Conacyt

**Catedrática Conacyt- Instituto Internacional de Estudios Políticos Avanzados, Universidad Autónoma de Guerrero, Candidata en el Sistema Nacional de Investigadores ConacyT, Consultora y coordinadora para la Unode en el estado de Guerrero jdonosoji@conacyt.mx

***Maestro en gobierno y asuntos públicos de la Facultad Latinoamericana de Ciencias Sociales con sede México, Consultor independiente para la ONUDC.
\end{abstract}

Cómo citar: Donoso, J., Antoine, P. (2018) Propuestas de políticas públcias para la prevención de la violencia y el delito en Acapulco, Guerrero 2018. Inciso 20 (1); 137-145.

Recibido: 15/04/2018Ｒevisado: 26/04/2018Ａceptado: 30/08/2018

\section{Resumen}

Este documento contiene una serie de recomendaciones de políticas públicas que pretenden contrarrestar los problemas de violencia e inseguridad que se viven en el reconocido destino turístico del estado de Guerrero, el puerto de Acapulco. Hoy, figura como una de las ciudades más peligrosas del mundo. Para el diseño de estas recomendaciones se utilizaron los resultados del diagnóstico participativo del proyecto de Fortalecimiento de políticas públicas en materia de seguridad y justicia en Guerrero elaborado en 2017-2018 por la oficina para la Droga y el Delito de la Organización de las Naciones Unidas (Unodc) con sede en México, las cuales tienen mucha relación con los Objetivos para el Desarrollo Sostenible (ODS). El diagnóstico se realizó tomando en cuenta la participación de diversos actores de la administración pública estatal y municipal, policías, custodios, organizaciones de la sociedad civil, sector empresarial y la población abierta.

Palabras clave: Violencia, inseguridad, políticas públicas, delitos,

\begin{abstract}
This article aims to provide a series of recommendations to counteract the violence in Acapulco, which is classified as one of the most dangerous cities in the world. They are based on the results of a diagnosis realized between 2017-2018 by the Office for Drugs and Crime of the Organization of the United Nations (UNODC) in Guerrero about: "Strengthening Public Policies tools in the Area of Security and Justice in Guerrero". We observed that the recommendations have strong similarity with some of the Sustainable Development Goals (SDG). The diagnostic has been realized with the participation of important actor such as: public administrator, law enforcement, prison officer, state government authorities, civil society organizations, the business sector and the population.
\end{abstract}

Keywords: Violence, insecurity, public policies, crimes, 


\section{Introducción (Antecedentes)}

Estas recomendaciones surgen como resultado del diagnóstico realizado en el proyecto de "Fortalecimiento de políticas públicas en materia de seguridad y justicia en Guerrero"'. Las recomendaciones son una síntesis de los principales desarticuladores ${ }^{2}$ de violencia discutidos entre los actores de la sociedad civil, institucionales y empresarios, y constituyen un primer avance para debatir la mejor combinación de acciones a impulsar para enfrentar de forma integral los problemas de inseguridad ${ }^{3}$ en uno de los municipios más importante del estado de Guerrero.

Acapulco, se encuentra sumido en una espiral de violencia que no parece tener punto de retorno, difícil de abordar y con un carácter multidimensional, por lo que es importante utilizar una metodología que permite la interacción entre los participantes del proyecto. Para eso, se estableció un diálogo entre los distintos actores de la vida social; promoviendo la confianza y participación para expresar libremente sobre los factores que expliquen la violencia así como las posibles acciones a considerar.

Una vez realizado, las mesas de trabajo fueron analizadas y de ahí se extrajeron todos los conceptos explicativos de la violencia y los elementos de respuesta. Adicionalmente, se elaboró una encuesta basada en ocho dimensiones (Bienestar subjetivo, cohesión social, las libertades, aspectos de violencia estructural, corrupción y honestidad, la procuración de

\footnotetext{
1. Este estudio fue financiado por el gobierno del estado de Guerrero con fondos del Programa Nacional para la Prevención Social de la Violencia y el Delito (Pronapred) y ejecutado por la Unodc. El diagnóstico se realizó en seis municipios del estado de Guerrero: Acapulco, Chilpancingo, Chilapa, Cocula, Iguala, Zihuatanejo durante los meses de octubre de 2017 y abril de 2018.

2. Una de las tres categorías analíticas y conceptos creados para este diagnóstico. Se entiende como las medidas adoptadas para generar estrategias de transformación en la conducta social e institucional violenta. Operan como una agencia dual, ya que puede ser promovida tanto por las instituciones como por la sociedad civil y eso quiere decir que son inhibidores de la violencia estructural y contingente. Los desarticuladores de la violencia son funcionales y transforman conductas y relaciones sociales.

3. A veces se usa el concepto de inseguridad para evitar el uso excesivo del concepto de violencia, pero no se considera un sustituto en el sentido estricto de la palabra.
}

justicia, aspectos de violencia contingente, desarticuladores de violencia y las características socioeconómicas) para buscar generalizar los conceptos aprendidos, transitando de un estudio cualitativo a uno cuantitativo (Morgan, 1998) . Con la información validada de las mesas de trabajo a través de la encuesta, se diseñaron las recomendaciones de políticas públicas.

\section{Contexto}

El caso de Acapulco como objeto de estudio es complejo, porque es una de las ciudades más peligrosas del país y del mundo, de acuerdo con el estudio Ranking de las 50 ciudades más violentas del mundo 2017 del Consejo Ciudadano para la Seguridad Pública y Justicia Penal,. Apoyados en la revisión de prensa del periódico El Sur durante el año $2017^{4}$, se encontró que el municipio presenta 799 homicidios aproximadamente, situación que lo posiciona como el número uno en muertes prevenibles en el estado de Guerrero con $40.8 \%$.

De estos, $88 \%$ de las víctimas son hombres y $8 \%$ mujeres, la edad promedio de las muertes es de 33 años. Otro dato interesante en el análisis de prensa es que Acapulco es el municipio con la mayor cantidad de cuerpos reportados en fosas clandestinas con un total de 43. Asimismo, cuando se observa el mapa de la violencia, se encuentra, que a pesar de existir programas como Pronapred, cuyo objetivo es reducir la violencia desde un enfoque social, los lugares con el mayor número de homicidios en Acapulco son las consideradas por Pronapred (ver figura 1), es decir, Progreso, Las Cruces, Renacimiento, Centro, Emiliano Zapata, Miguel Alemán, La Laja, Morelos, Unidad Habitacional El Coloso y la Sinaí.

4. Lo relevante de la revisión de prensa es que el monitoreo permite ver cuáles son los casos de muertes con mayor impacto mediático y social, así como el acceso y visibilidad de estos episodios violentos hacia la ciudadanía, lo que nos permitió dimensionar la exposición social de la muerte en el estado de Guerrero. Por otro lado, el monitoreo contribuye a indagar en la morfología y la multiplicidad de expresiones violentas a las que se encuentran expuestas las personas de cada municipio, algo que los datos duros no nos posibilitan observar. 
Figura 1. Delitos más frecuentes en los polígonos de Acapulco

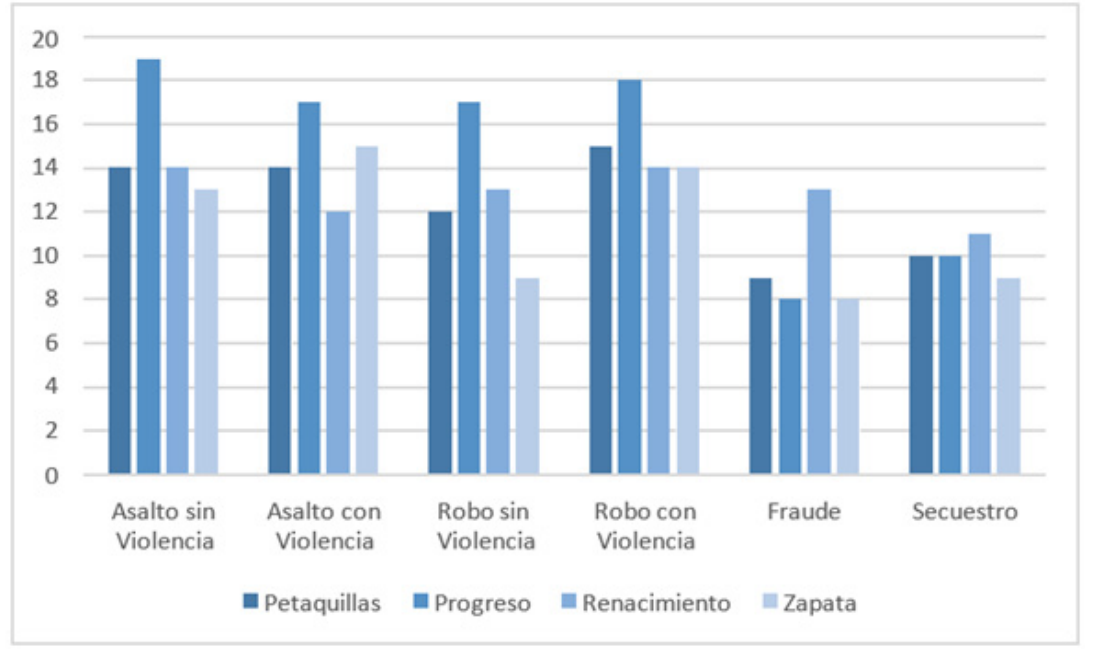

Fuente: Elaboración propia con datos de la Encuesta sobre medición de la violencia en el estado de Guerrero, 2018

De acuerdo con los resultados de las mesas de trabajo con autoridades (ver figura 2), sociedad civil y empresarios, se cree que los problemas de seguridad pública son generados por la pobreza. En este sentido es interpretado como un factor que alimenta la delincuencia y la violencia.

Por otra parte, la corrupción institucional en su completa magnitud es referida como un 'cáncer' que obstaculiza el buen funcionamiento de la justicia y sus organismos operativos para inhibir el delito y disminuir la violencia. La inseguridad, los homicidios y la extorsión son los problemas más graves de seguridad con que se enfrenta Acapulco.

Figura 2. Víctimas de algún delito en los polígonos de Acapulco

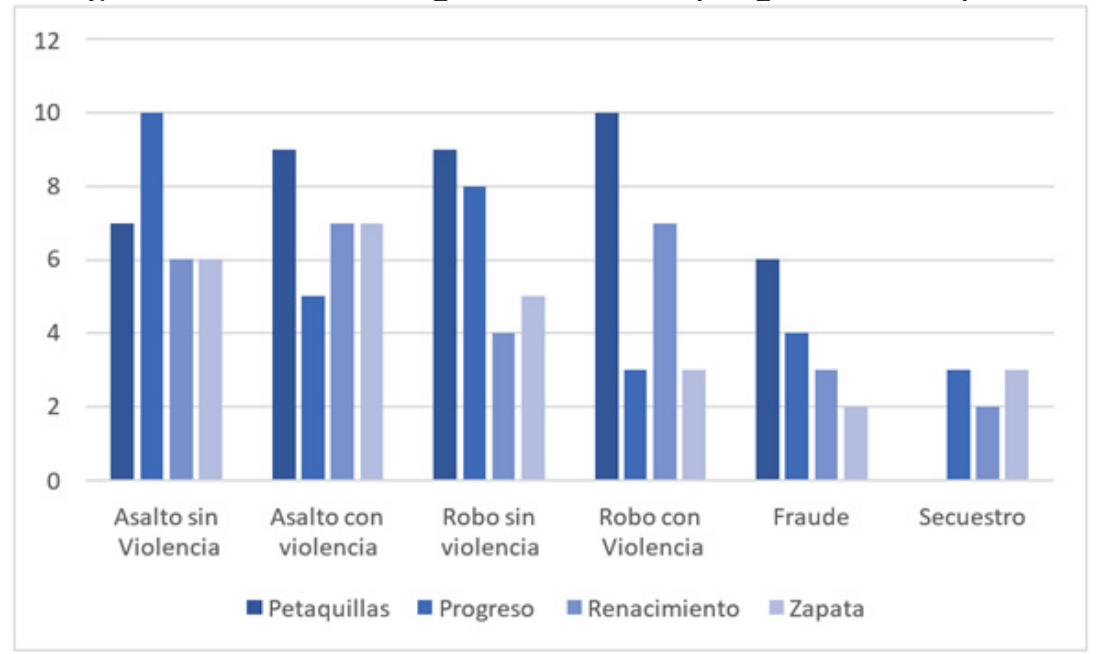

Fuente: Elaboración propia con datos de la Encuesta sobre medición de la violencia en el estado de Guerrero, 2018

\section{INCIST}


La Encuesta de percepción de la violencia en el estado de Guerrero se realizó en cinco polígonos: Zapata, Renacimiento, Progreso, Petaquillas y Llano Largo. Se contempló aplicarse en la colonia Jardín, pero debido a las condiciones de seguridad, se sustituyó por Llano Largo. En el municipio de Acapulco se levantaron 168 encuestas, de las cuales 122 corresponden a estos polígonos, el resto se aplicó en otras colonias consideradas de medio y alto ingresos, con el fin de identificar diferencias en la percepción de la violencia y asegurar la validez del instrumento. Considerando cinco polígonos del municipio para responder la siguiente pregunta ¿Usted conoce o sabe por sus vecinos de los delitos más frecuentes en su localidad? Observamos lo siguiente en la figura 3:

Figura 3. Delitos más frecuentes en Acapulco

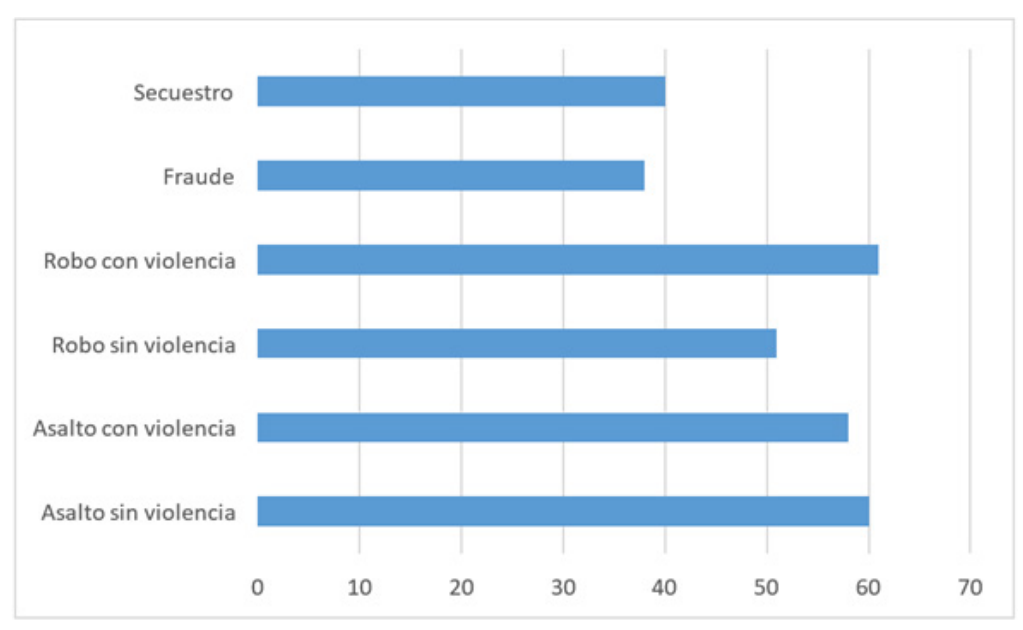

Fuente: Elaboración propia con datos de la Encuesta sobre medición de la violencia en el estado de Guerrero, 2018

Con respecto a la siguiente pregunta Dígame si usted ha sufrido de algún tipo de violencia en su localidad, los resultados fueron los siguientes expresados en la figura 4:

Figura 4. Victiación directa en los polígonos de Acapulco

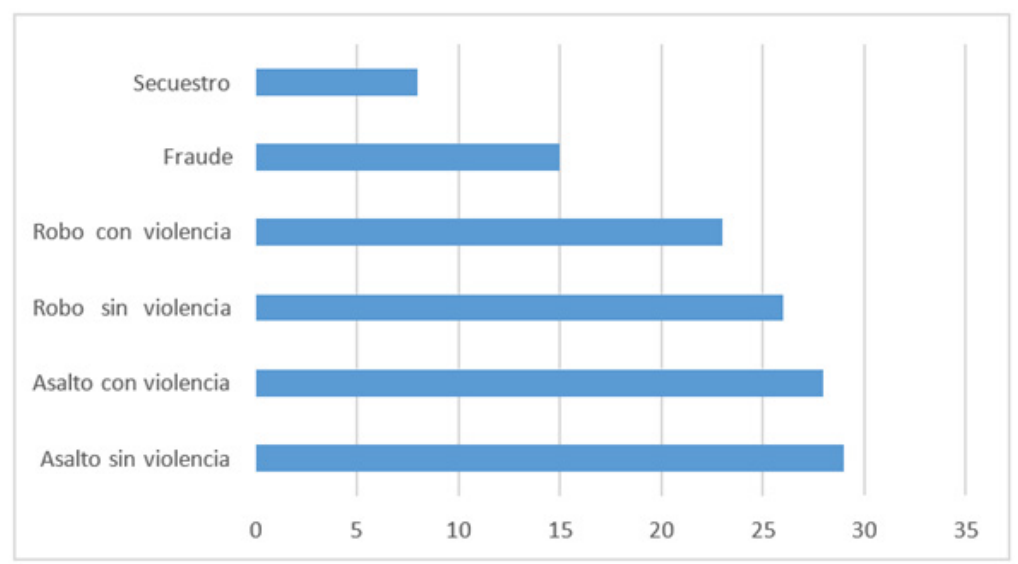

Fuente: Elaboración propia con datos de la Encuesta sobre medición de la violencia en el estado de Guerrero, 2018 
Aunque los polígonos de Petaquillas y Progreso tienen el número de homicidios más alto, de acuerdo con el monitoreo de prensa, la percepción de victimización se distribuye en primer lugar en Petaquillas, Progreso y Renacimiento. Una de las explicaciones de esta violencia es que en estos polígonos se concentra la mayor actividad comercial de la ciudad, por lo que la comisión de delitos tiene que ver con la extorsión y el cobro de cuotas. Es importante considerar que es en Petaquillas donde se encuentran los dos mercados más grandes que abastecen a la ciudad de todo tipo de bienes, lo que nos permite visualizar que gran parte de los homicidios cometidos en el puerto de Acapulco están relacionados con delitos vinculados con células criminales que viven de la extorsión y no necesariamente con la venta de drogas, como se presume generalmente. Es a los comerciantes a quienes acosó la actividad criminal en el puerto durante el año 2017 y les provocó una asfixia financiera, especialmente a los pequeños y medianos. Lo anterior pretende demostrar que es necesario replantearse el concepto de polígono propuesto por el Pronapred, ya que la inseguridad y violencia a la que se encuentra sometida la sociedad acapulqueña no está relacionada única y exclusivamente con su condición de pobreza y marginalidad, sino que es una situación generalizada que trasciende los estratos socioeconómicos en términos operativos y de victimización.

\section{Cambio de paradigma}

A través de este diagnóstico, aprendimos que es fundamental repensar el análisis de violencia. El combate a la violencia, la criminalidad o la delincuencia, requiere ser visto desde un enfoque de justicia social, equidad de oportunidad para poder sanear la deuda histórica con el pueblo de Acapulco y el estado de Guerrero.

El planteamiento propuesto consiste en cambiar la forma de pensar el desarrollo de las personas en función de sus libertades, la dignidad humana y de manera sostenible, reorientar las políticas para centrarlas en los individuos. Así, como fue señalado por el Departamento de Asuntos

Económicos y Sociales de la Naciones Unidas, la igualdad de oportunidades se manifiesta cuando la diferencia que existe entre los individuos depende únicamente de ellos y no por las desventajas atribuidas a factores que no tienen bajo control(Un/desa, 2015) . La justicia, en este sentido, es un atributo deseable de la sociedad y por esa misma razón, su consecución es responsabilidad del Estado. Si la definición de los grupos socialmente excluidos y las libertades que les son restringidas implica un particular uso del poder, el problema de la exclusión social requiere de decisión y acción colectiva. Es decir, es un problema de gobierno. El concepto de justicia social es central a las normas éticas para evaluar lo óptimo de una situación. El enfoque de capacidades puede ser definido como un marco de evaluación comparativa de calidad de vida y de teorización sobre justicia social. Sostiene que la pregunta clave al comparar sociedades y evaluarlas en función de la justicia es “¿Qué puede hacer y ser cada persona?”. En otras palabras, el enfoque toma a cada persona como un fin, no inquiriendo sólo sobre el nivel total o promedio de bienestar, sino sobre las oportunidades disponibles para cada persona.

\section{Recomendaciones de políticas públicas}

El estudio arroja importantes cuestionamientos sobre las dimensiones de violencia, no solo para próximas investigaciones, sino revela áreas a considerar en la discusión sobre las políticas de combate a la inseguridad. Los temas abarcan una gran serie de dimensiones sociales, políticas y económicas que para fines de las recomendaciones agruparemos en ejes temáticos que coinciden de manera armónica con las propuestas impulsadas por la Organización de las Naciones Unidas a través de los ODS 2030. 


\section{Igualdad de oportunidades}

El primer eje procura acciones para reducir la pobreza y la desigualdad en el puerto de Acapulco. Si bien estos puntos pudieran considerarse como elementos no directamente relacionados con la violencia y la criminalidad, son unas de las principales preocupaciones y temas de las mesas de trabajo. Estos elementos fueron abordados de distintas formas entre los participantes: marginación, falta de recursos, dinero, carencias, salario digno, desventaja, oportunidades, entre otros; los cuales fueron identificados como las causas estructurales, históricas y sociales de la situación actual de crisis de inseguridad “... la pobreza ha hecho que los mismos padres estén de acuerdo con lo que hacen, dicen: bueno, antes no había qué comer y ahora tenemos qué comer..." (Mesa de trabajo con empresarios, 5 de diciembre de 2017). Es de recordar que hay una fuerte correlación entre pobreza y violencia, que se manifiesta por la necesidad de contar con recursos suficientes para adquirir los bienes y servicios que se requieren para satisfacer sus necesidades (alimentarias y no alimentarias)

“... la pobreza, desgraciadamente, es uno de los factores prioritarios por los cuales, mucha gente que no tiene una forma de llevar un sustento a su familia y si le ofrecen vehículos, armas y le van a pagar bien, es candidato a delinquir..." (Mesa de trabajo con autoridades del gobierno municipal, Acapulco, 20 de noviembre de 2017).

Para eso, se propone lo siguiente:

- Creación de un fondo para repartir a las familias de los primeros 5 deciles de ingreso: dicho fondo consiste en ayudar a las familias con los gastos básicos, especialmente adquisición de comida para el hogar, garantizando la seguridad alimentaria de las familias. En esta etapa, se requiere empujar esta iniciativa a través los mecanismos del congreso local y paralelamente trabajar con los empresarios y la sociedad civil sobre la implementación de dicha recomendación. Solo se necesita de voluntad política.

- Promover lafocalización de los programas de combate a la pobreza: uno de los principales problemas de los programas de combate a la pobreza en México es la focalización, con este antecedente se requiere de la acción por parte del gobierno del estado para proceder a la reevaluación de los programas federales y locales para reducir la pobreza.

- Promover el salario digno de los trabajadores: un mejor salario implica mayor incentivo para los trabajadores, mayor compromiso con sus actividades y menor probabilidad de ponerse al servicio de la delincuencia.

\section{Estado de derecho}

Instaurar un estado de derecho en Acapulco es fundamental para el buen funcionamiento de la sociedad y la construcción de la paz. Según Sergio Márquez (2015), el derecho es la fuerza coercitiva del Estado que reprime las desviaciones, los incumplimientos y resuelve las controversias que se presenten dentro del amplio pacto social. Este concepto abarca un sinnúmero de ámbitos para la acción de gobierno como: el combate a la corrupción y la impunidad, promoción de la justicia, presencia del estado sobre el territorio, el respeto a las normas, entre otras. “... hay algo muy importante que considero que hay que combatir, y es el principal precursor de la violencia en nuestro estado y de Acapulco: la corrupción..." (Mesa de trabajo con autoridades municipales, 20 de noviembre de 2017).

En efecto, la nueva Agenda de los ODS reconoce la necesidad de construir sociedades pacíficas, justas e inclusivas que proporcionen igualdad de acceso a la justicia y se basen en el respeto de los derechos humanos (incluido el derecho al desarrollo), en un estado de derecho efectivo 
y una buena gobernanza a todos los niveles, y en instituciones transparentes y eficaces que rindan cuentas (International Development Law Organization, 2018)

En este sentido, el panorama en el estado de Guerrero en cuanto a los indicadores de corrupción y de justicia deja mucho que desear ya que “... la mala procuración de los penales propicia que estos se vuelvan escuelas del crimen organizado..." (Mesa de trabajo con empresarios, 5 de diciembre de 2017).

Para eso, se propone lo siguiente:

- Recuperación del control territorialpor parte de los pobladores y comunidades: el control territorial es caracterizado por ser uno de los pilares en la lucha contra la delincuencia, como también para la promoción de mayor bienestar a la población. Esto implica conocer cómo se generan los conflictos en el territorio, la naturaleza de los problemas sociales que enfrenta la población, así como las alianzas con que cuenta el gobierno para impulsar los cambios esperados.

- Focalizar las acciones de construcción de redes viales en zonas marginadas: estas permiten la libre circulación de los bienes y servicios, por lo cual es imperativo asegurar el acceso a redes viales seguras a las poblaciones marginadas.

- Establecer un sistema de transporte público-privado eficiente: el sistema de transporte es caracterizado por ser uno de los medios cooptado por la delincuencia. Se sugiere un sistema controlado por la iniciativa pública-privada.

- Transparencia y rendición de cuentas: es el sistema por el cual se informa sobre la realización de sus actividades. También, se le reconoce por sus virtudes en el combate a la corrupción, la irresponsabilidad, la negligencia, la deshonestidad, así como cualquier acto inmoral de los gobiernos. Promover la transparencia y rendición de cuentas implica hacer partícipe a la sociedad civil, informándola sobre la ejecución de los recursos.

- Procuración de justicia: La falta de acceso a la justicia implica que los conflictos quedan sin resolver y que las personas no pueden obtener ni protección ni reparación. Las instituciones que no funcionan con apego a la ley son propensas a la arbitrariedad y al abuso de poder, y tienen menos capacidad para prestar servicios públicos para todos ${ }^{5}$; asimismo, se puede observar que la percepción de la población en cuanto a la procuración de justicia por parte de las instituciones es muy baja. Así que, para lograr la paz, la justicia y la inclusión, es importante que los gobiernos, la sociedad civil y las comunidades trabajen juntos para poner en práctica soluciones duraderas que reduzcan la violencia, hagan justicia, combatan eficazmente la corrupción y garanticen en todo momento la participación inclusiva.

- Construcción de cárceles con sentido social: la construcción de cárceles con sentido social ha sido uno de los temas redundantes en las mesas de trabajo con los privados de libertad. Muchos de ellos reconocen estar ahí por la falta de oportunidad; sin embargo, esperan que al menos la cárcel sea un espacio de reinserción y no un medio para seguir delinquiendo.

2. Eficiencia de la administración pública

La eficiencia ha sido uno de los temas controversiales cuando se refiere a la administración pública, ya que, en general se

5. https://www.un.org/sustainabledevelopment/es/wp-content/uploads/sites/3/2017/01/ Goal_16_Spanish.pdf consultado el 6 de mayo de 2018 
reconoce a las instituciones públicas por ser ineficientes y lentas “... el exceso de trabajo, las tareas pendientes, las persecuciones, además de los malos horarios y descanso no respetados, crea que salga toda nuestra ira y no trabajemos adecuadamente..." (Seminario con policías, 23 de noviembre de 2017)

En medio de las posiciones teóricas, se admite la necesidad de contar con un aparato administrativo eficiente, profesional y capacitado que pueda usar los instrumentos de políticas públicas para lograr los objetivos planteados. En resumen, a fin de avanzar en la consecución de las recomendaciones, necesitamos instituciones públicas eficaces e inclusivas que puedan proporcionar con mayor igualdad los servicios públicos a todos. Sin embargo, es importante comprender, que la inversión debe ser integral, ya que se requiere perfeccionar el capital humano, pero también aspectos vinculados al equipamiento e infraestructura, principalmente en el área de seguridad pública “... dormimos en las patrullas, en las calles, donde sea y eso el Gobierno o los jefes no lo ven, donde nos agarra la noche ahí nos dormimos, medio dormimos un ratito, en los cuarteles no hay camas, no hay aire acondicionado, no hay nada, duerme uno donde se pueda ..." Seminario con policías, Acapulco, 23 de noviembre de 2017.

Para eso, recomendamos lo siguiente:

- Profesionalización de la administración pública: aun cuando existen varios programas de capacitación para los funcionarios, siguen existiendo dudas sobre su efectividad. Los mismos trabajadores han expresado sus inquietudes sobre dichos programas y la necesidad de contar con una profesionalización eficiente (ver anexo gráfico 1 y 2), especialmente en el caso particular de Acapulco.
- Mejoramiento de las condiciones laborales: va de la mano con la profesionalización. Para que los funcionarios puedan realizar en tiempo y forma sus labores necesitan contar con los medios necesarios para hacerlo.

- Gobierno Digital: el uso de la tecnología de la información permite mejorar la productividad y reducir gastos innecesarios.

- Sistema de monitoreo y evaluación de políticas públicas: Los sistemas de Monitoreo y Evaluación (MyE) son fundamentales para el éxito de las políticas públicas, numerosas publicaciones, consultorías $\mathrm{y}$ artículos científicos han abordado de manera contundente su importancia al momento de diseñar e implementar las políticas públicas.

- Fortalecimiento y empoderamiento de grupos vulnerables: mujeres, jóvenes y niños Empoderar económicamente a los grupos vulnerables es sin duda una de las medidas que impactan directamente en la pobreza y la desigualdad e incrementan la participación de la población en el crecimiento económico. Sin embargo, es importante tomar en cuenta medidas efectivas que aseguren la creación y acumulación de riqueza de los grupos vulnerables para evitar posibles efectos contradictorios de esta política.

Para eso, proponemos lo siguiente ${ }^{6}$ :

- Apoyar la iniciativa empresarial y el desarrollo de empresas de los grupos vulnerables, lo que incluye el acceso de los grupos vulnerables a la financiación, a los mercados y a otros recursos que aumenten su capacidad productiva y su competitividad.

- Apoyar a las empresas del sector social mediante el fortalecimiento de las

6. Propuestas adaptadas a partir de la siguiente fuente: http://www.undp.org/content/dam/ undp/library/corporate/fast-facts/english/FF- Economic-Empowerment-of-Youth-SP.pdf consultado el 7 de mayo de 2018. 
capacidades de los grupos vulnerables para ayudar a las comunidades, resolver problemas sociales, aumentar el empleo entre la población, reducir la pobreza y devolver dividendos a la comunidad.

- Trabajar con los sectores público y privado con miras a fomentar pasantías y programas e incentivos de aprendizaje entre los empleadores privados y públicos, para que contraten a los grupos vulnerables o sus servicios.

\section{Conclusiones}

A modo de conclusión, es importante comprender la violencia como un fenómeno social multidimensional por lo que las soluciones deben procurar atacar las causas y no solamente los síntomas. El combate contra la inseguridad $\mathrm{y}$ violencia ha tendido a segmentar aún más la población y las ciudades, estigmatizar a ciertos grupos de pobladores y victimizar a otros, invisibilizando a sectores significativos como jóvenes, mujeres y niños.

Las fallidas políticas del combate contra el narcotráfico en México en los últimos 12 años se han volcado principalmente en la militarización $\mathrm{y}$ en la prevención del delito combinada con una progresiva carrera armamentista, lo que nos obliga a pensar en soluciones más integrales, que estén enfocadas en las particularidades locales y las problemáticas específicas para buscar soluciones a la medida.

El diagnóstico elaborado por la Onudc para el estado de Guerrero durante el año 2017 nos proporciona un análisis que profundiza en los principales temas que preocupan a los diversos actores de la sociedad acapulqueña, que fueron discutidos a lo largo de este diagnóstico. Comprender de manera social, política y cultural la violencia en el puerto de Acapulco nos permite observar que la igualdad de oportunidades, el estado de derecho, la eficiencia de las instituciones públicas y el empoderamiento económico son temas estratégicos para reducir la incidencia delictiva y los índices de homicidios en el puerto.

\section{Referencias bibliográficas}

Márquez, S. (2008) Estado de derecho en México. En Cienfuegos, D., Rodríguez, L. (coords) Estado, Derecho y Democracia en el momento actual. 212- 203. https:// archivos.juridicas.unam.mx/www/bjv/ libros/6/2990/13.pdf

Morgan, D. L. (1998). Practical Strategies for Combining Qualitative and Quantitative Methods: Applications to Health Research. Qualitative Health Research, 8(3), 362-376. doi:10.1177/104973239800800307

Organización de las Naciones Unidas.Paz,justicia e instituciones sólidas (2017). En https:// www.un.org/sustainabledevelopment/ es/wp-content/uploads/ sites/3/2017/01/ Goal_16_Spanish.pdf

El estado de derecho y la agenda 2030: El camino al futuro. Nota introductoria para un debate interactivo. (2015) En https://www.idlo.int/system/files/ event-documents/AP2015- 6.1R-Nota-informativa-debate-interactivo-agenda-2030-IDLOAsamblea2015 0.pdf

Un/desa (2015) Concepts of Inequality in Development En: http://www.un.org/en/ development/desa/policy/wess/wess dev_issues/dsp_policy_01.pdf.

United Nations Development Programme (2017). Empoderamiento económico de la juventud. En http://www.undp.org/ content/dam/undp/library/corporate/ fast-facts/english/FF- Economic-Empowerment-of-Youth-SP.pdf 